\title{
Late onset presentation of Leigh syndrome on two cases
}

\author{
Ursula Thomé ${ }^{1}$, Alexandre Fernandes², \\ Osvaldo José Moreira do Nascimento ${ }^{3}$, \\ Marcos Raimundo Gomes de Freitas ${ }^{4}$, \\ Marco Antônio Araujo Leite 5
}

Leigh's syndrome (LS) or subacute necrotizing encephalomyelopathy, described by Leigh in 1951, is characterized by symmetrical necrotic lesions in the central nervous system, involving areas such as the basal ganglia, brainstem, spinal cord and cerebellum. It is a mitochondrial disease related to various enzymatic defects that affect the oxidative metabolism ${ }^{1}$. It affects mainly infants and preschool children. But its presentation is extremely variable and may occur in children and young adults in atypical way ${ }^{2,3}$. The most common symptoms are motor with pyramidal and cerebellar features ${ }^{1,4,5}$. Beside this, the disease has characteristic findings on anatomopathological examination, neuroimaging and cerebrospinal fluid (CSF) examination ${ }^{1,4}$. The treatment effectiveness is still controversial, and aims to decrease the anaerobic metabolism and consequently the production of free radicals ${ }^{1}$.

We analyze two cases of LS.

\section{CASES}

\section{Case 1}

A 10-year-old boy, born from a non consanguineous couple, had a history of having started changing in gait. He began to make involuntary movements with the right foot and leg, which worsening after a month, affecting the upper limb on the same side. It were noted, specially when he walked or maked flexion of the wrist and fingers. After four months, the left side was also involved. The patient evolved with progressive worsening in walking, frequent falls, no longer write or eat alone or perform personal hygiene care such as bathing or brushing teeth by himself.

At this time, physical examination showed dystonic movement in the 4 limbs, more severe on the right side. His strengh, tone or reflexes were normal. The fundus oculi and the slit lamp examination were both normal.

None alterations were seen in the hemogram, biochemistry (including serum lactate), ceruloplasmin, copper or CSF examination. The genetic research for Huntington's disease (assessment of CAG repeat) and LS (mutation 8993) were negative. A biopsy of muscle did not shown the red ragged fibers. The magnetic resonance imaging (MRI) and spectroscopy (S-MRI) of the brain showed symmetrical hyperintense signal in the putamen on T2-weighted sequences, associated with the lactate's peak (Fig 1).

\section{Case 2}

A 6-years-old girl, born from a non consanguineous couple had a history of having a cold. After three days the onset of symptoms, she presented weakness of lower limbs, fever and drowsiness. At this
Correspondence

Ursula Thomé

Rua Siqueira Campos 53 / 1204 22031-070 Rio de Janeiro RJ - Brasil

E-mail: ursulathome@yahoo.com

Received 28 May 2009

Received in final form 6 August 2009

Accepted 17 August 2009

\section{APRESENTAÇÃO TARDIA DA SÍNDROME DE LEIGH: A PROPÓSITO DE DOIS CASOS}

Department of Neurology, Antonio Pedro University Hospital, Federal Fluminense University, Niterói RJ, Brazil: 'MD, Neuropediatrician, Student of Post-Graduation Program in Neurology and Neuroscience in Federal Fluminense University (UFF): ${ }^{2} \mathrm{MD}, \mathrm{MSc}$, Neuropediatrician, Professor of Pediatrics in Federal Fluminense University (UFF); ${ }^{3} \mathrm{MD}, \mathrm{PhD}$, FAAN, Neurologist, Chair in Neurology. Coordinator of the Post-Graduation Program in Neurology and Neuroscience in Federal Fluminense University (UFF); ${ }^{4} \mathrm{MD}$, PhD, Neurologist, Chair in Neurology. Professor of Post-Graduation Program in Neurology and Neuroscience in Federal Fluminense University (UFF); ${ }^{5} \mathrm{MD}$, MSc, Neurologist of Antonio Pedro University Hospital (UFF). Professor of Neurology in UNIGRANRIO's Scholl of Medicine. 


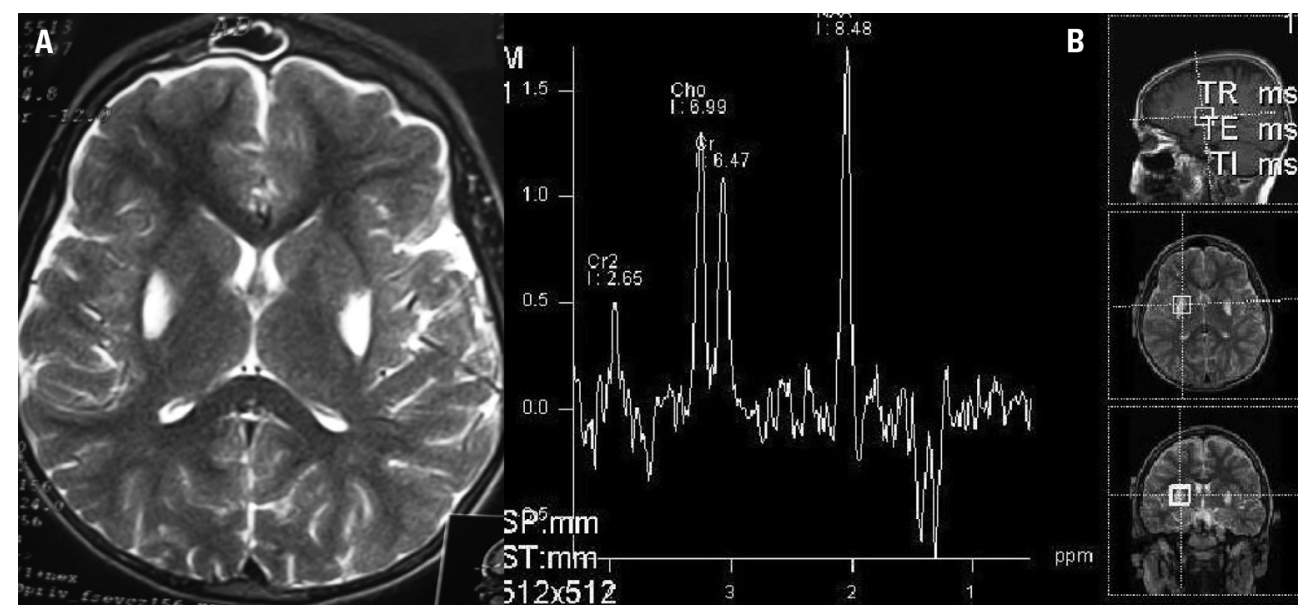

Fig 1. MRI depicting increased signal change within putamen bilaterally in Patient 1 [A] and S-MRI showing lactate peak [B].

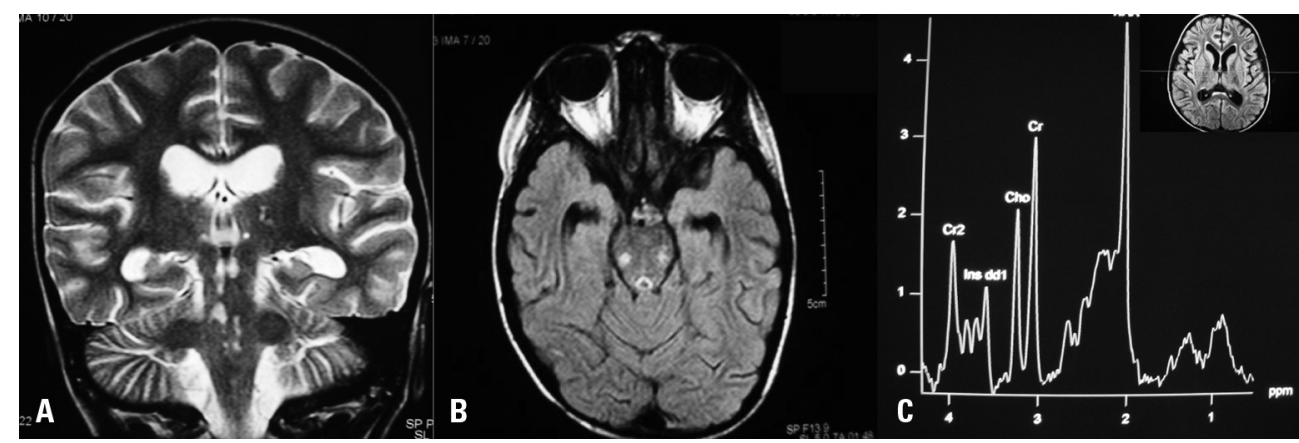

Fig 2. Case $2 \mathrm{MRI}$ and S-MRI showing bilateral lesions on both thalamus, pons, medulla, the topography of the cortico-spinal tract and the median longitudinal fasciculus [A], mesencephalon, periaqueductal gray matter [B] and the characteristic lactate peak in 1.3 ppm [C].

time she was hospitalized and 24hs later, she became comatous and had respiratory failure.

On examination, the patient was comatous, tetraplegic and presented diffuse anesthesia.

None alterations were seen in the hemogram, biochemistry and CSF (including the biochemistry, the bacterioscopy and the culture). The PCR for enterovirus and HSV (in the CSF) were negative. CSF lactate $=1.3 \mathrm{nmol}$. Serum lactate $=1.5 \mathrm{nmol}$. The MRI and S-MRI of brain showed hypointense signal on T1-weighted sequences and hyperintense in other sequences, involving the periaqueductal gray matter, both thalamus (in a symmetric way), mesencephalon, pons, medulla, the topography of the cortico-spinal tract and the median longitudinal fasciculus, associated with the lactate's peak on S-MRI (Fig 2).

After 1 month of hospitalization in the Intensive Care Unit, she remained with the same neurological symptoms and started to improve her motor responses, and respiratory drive. There was a slow and gradual improvement of neurological symptoms, specially in motion and awareness. Later, she began to walk and started making sounds that were gradually being replaced by full speech. In this period, it was noted the paralisis of the III and VI cranial nerve and bilateral ptosis.

This research project has been approved by the ethics committee of our Institute of Neurology and conforms to the provisions of the Declaration of Helsinki in 1995 (as revised in Edinburgh 2000).

The patients agree with the submission of their magnetic resonance image and the informed consent document was signed by their parents.

\section{DISCUSSION}

Although rare, LS is probably the most common mitochondrial disease in childhood ${ }^{5}$. There are 2 forms of presentation: infant and juvenile. In the infantile form (about $50 \%$ of cases), symptoms may become apparent within two years of life. In this situation, there may be hypotonia, regression of neuropsychomotor development, ataxia, seizures and breathing disfunctions ${ }^{1,5}$. On the juvenile form which is much less common, patients have mainly an extrapyramidal syndrome with dystonia and stiff- 
ness. There are no specific markers for this disease, and the lactate's elevation in serum and CSF may not be detected, especially when the patient is not under stress of oxidative metabolism ${ }^{1}$. Its etiology is genetic and is related to an enzyme defect in any part of the cell respiration. The most common defects are biochemical deficiencies of pyruvate dehydrogenase complex, complex IV (or cytochrome $\mathrm{C}$ oxidase), in the complex I of respiratory chain or in the ATPase ${ }^{5-7}$.

The genetic inheritance of LS may be autosomal recessive, X-linked or mitochondrial ${ }^{6}$. As the sensitivity of genetic tests available is low, in most cases this diagnosis is suspected by MRI, especially in late or atypical forms ${ }^{1,8}$. The typical image of LS consists of symmetric areas of hyperintense signal on T2 in the basal ganglia, cerebral peduncles and periaqueductal region. It is believed that the preference for such sites is due to an increased metabolic activity in these areas, which are more sensitive to ATP's synthesis failure. The S-MRI has been of great value in this syndrome, for showing lactate's peak in the affected areas ${ }^{8,9}$.

The two cases described represent atypical forms of presentation of this disease. In case 1, the patient has the onset of symptoms only at the age of 10 years old, characterizing the late form of presentation. Its first manifestation was only the dystonia and the evolution of neurological symptoms was slow and gradual, instead of bursts. In case 2, the symptoms of encephalopathy and ophthalmoplegia appeared after a respiratory infection, as the typical installation of the disease (after a metabolic stress). However, like the first case, the onset of symptoms occurred later, when the patient was 6 years old. But her evolution was different, characterized by periods of exacerbation and remission of neurological symptoms. The two cases illustrate how the patients with this disease may progress in outbreaks or a slow and gradual progression $^{1-3}$. This slow progression seems to occur more frequently in the late form of presentation ${ }^{2,3}$.

These diagnoses were made by the association of clinical manifestations with the findings on MRI. In both cases the mitochondrial mutation seen as more common (T8993C) was negative, which does not exclude the diagnosis, due to the wide possibility of mutations described ${ }^{10}$.

The mitochondrial diseases have emerged as a challenging, specially in terms of diagnosis, evolution and treatment. The development on molecular and biochemical tests in the recent decades has discovered many enzymatic defects responsible for this syndrome. Greater knowledge of this entity, might improve the prognosis of these patients, changing the natural history of this disease as well the genetic counseling in prenatal period.

\section{REFERENCES}

1. Fernandes AR. Uma revisão clínico-laboratorial da síndrome de Leigh baseada em 7 casos (Dissertação). Rio de Janeiro: Fundação Oswaldo Cruz - Instituto Fernandez Figueira. Departamento de Genética, 2001.

2. Huntsman RJ, Sinclair DB, Bhargava R, Chan A. Atypical presentations of Leigh syndrome: a case series and review. Pediatr Neurol 2005;32:334-40.

3. Berkovic SF, Karpati G, Carpenter S, Lang AE. Progressive dystonia with bilateral putaminal hypodensities. Arch Neurol 1987;44:1184-1187.

4. Wick R, Scott G, Byard RW. Mechanisms of unexpected death and autopsy findings in Leigh syndrome (subacute necrotising encephalomyelopathy). J Forensic Leg Med 2007;14:42-45.

5. Bhattacharya K, Rahman S, Leonard J. Diagnosis and management of mitochondrial respiratory chain disorders. Curr Paediatr 2003;13:536-542.

6. Absalon MJ, Harding CO, Fain DR, Li L, Mack KJ. Leigh syndrome in an infant resulting from mitochondrial DNA depletion. Pediatr Neurol 2001;24:60-63.

7. Graeber MB, Muller U. Recent developments in the molecular genetics of mitochondrial disorders. J Neurol Sci 1998;153:251-263.

8. Haas R, Dietrich R. Neuroimaging of mitochondrial disorders. Mitochondrion 2004;4:471-490.

9. Takahashi S, Oki J, Miyamoto A, Okuno A. Proton magnetic resonance spectroscopy to study the metabolic changes in the brain of a patient with Leigh syndrome. Brain Dev 1999;21:200-204.

10. DiMauro S, Hirano M. Mitochondrial encephalomyopathies: an update. Neuromuscul Disord 2005;15:276-286. 